\title{
Coffee consumption and risk of rare cancers in Scandinavian countries
}

Marko Lukic ${ }^{1}$, Lena Maria Nilsson², Guri Skeie ${ }^{1}$, Bernt Lindahl ${ }^{3}$, Tonje Braaten ${ }^{1}$

${ }^{1}$ Department of Community Medicine, Faculty of Health Sciences, UiT The Arctic University of Norway, Tromsø, Norway.

${ }^{2}$ Department of Public Health and Clinical Medicine, Nutritional Research, Umeå University, Umeå, Sweden

${ }^{3}$ Department of Public Health and Clinical Medicine, Occupational and Environmental Medicine, Umeå University, Umeå, Sweden

\section{Corresponding author:}

Marko Lukic

marko.lukic@uit.no

Institutt for Samfunnsmedisin,

UiT Norges Arktiske Universitet, N - 9037 Tromsø, Norway

Tel: +47 776 20823; Mobil: +47 40591581

\section{Key words:}

coffee, filtered, boiled, cancer, bladder, esophageal, kidney, pancreatic, stomach, prospective cohort study 


\begin{abstract}
Background Studies on the association between heavy coffee consumption and risk of less frequently diagnosed cancers are scarce. We aimed to quantify the association between filtered, boiled, and total coffee consumption and the risk of bladder, esophageal, kidney, pancreatic, and stomach cancers.
\end{abstract}

Methods We used data from the Norwegian Women and Cancer Study and the Northern Sweden Health and Disease Study. Information on coffee consumption was available for 193439 participants. We used multivariable Cox proportional hazards models to calculate hazard ratios (HR) with 95\% confidence intervals (CI) for the investigated cancer sites by category of total, filtered, and boiled coffee consumption.

Results Heavy filtered coffee consumers ( $\geq 4$ cups/day) had a multivariable adjusted HR of 0.74 of being diagnosed with pancreatic cancer (95\% CI 0.57-0.95) when compared with light filtered coffee consumers ( $\leq 1 \mathrm{cup} /$ day). We did not observe significant associations between total or boiled coffee consumption and any of the investigated cancer sites, neither in the entire study sample nor in analyses stratified by sex. We found an increased risk of bladder cancer among never smokers who were heavy filtered or total coffee consumers, and an increased risk of stomach cancer in never smokers who were heavy boiled coffee consumers. Conclusion Our data suggest that increased filtered coffee consumption might reduce the risk of pancreatic cancer. We did not find evidence of an association between coffee consumption and the risk of esophageal or kidney cancer. The increased risk of bladder and stomach cancer was confined to never smokers. 


\section{Introduction}

Many studies have aimed to clarify the mechanism(s) behind the biological effects of coffee components. Caffeine, the coffee diterpens kahweol and cafestol, and chlorogenic acid have been identified as the compounds that contribute most to the anticarcinogenic properties of roasted coffee (1-8). Of these compounds, the diterpenes deserve specific attention, since coffee brewing technique affects diterpene content to a large extent. Indeed, the diterpene content of boiled, unfiltered coffee may be as much as 60 times higher than that of filtered coffee (9).

In the past 2 decades, numerous studies have been conducted with the aim to quantify the association between coffee consumption and the risk of the most frequently diagnosed cancers. However, similar research that used rare cancer sites as the main outcome is less common. In a recently published meta-analysis by Wu et al, male coffee consumers and nonsmoking coffee consumers were found to have an increased risk of bladder cancer (10), whereas the International Agency for Research on Cancer concluded there was no consistent evidence of an association between drinking coffee and bladder cancer risk (11). On the other hand, the meta-analyses did not reveal any association between coffee consumption and the risk of esophageal, pancreatic, or stomach cancer (12-14). Similarly, the authors of the World Cancer Research Fund's second expert report found limited evidence of an association between coffee consumption and the risk of renal and pancreatic cancer $(15,16)$.

Norway and Sweden rank second and fourth in the world, respectively, in average per capita coffee consumption (17). We pooled the data from two Nordic cohorts: the Norwegian Women and Cancer (NOWAC) Study and the Northern Sweden Health and Disease Study (NSHDS), in order to explore the association between filtered, boiled, and total coffee consumption and the risk of bladder (worldwide age-standardized rate (ASR) per 100000 - 
5.3), esophageal (worldwide ASR - 5.9), kidney (worldwide ASR - 4.4), pancreatic (ASR 4.2) and stomach (worldwide ASR - 12.1) cancer (18).

\section{Methods}

\section{Participants}

We included participants from the NOWAC Study and the NSHDS. The data from these studies were merged into one dataset, which was used in the present paper. Detailed information on these two cohorts are available elsewhere $(19,20)$. In short, the NOWAC study was started in 1991 and consists of random samples of 172000 Norwegian women aged 30-70 years that completed a questionnaire regarding their lifestyle, diet, and health status (overall response rate: 52.7\%). Follow-up information was also collected on two occasions, with approximately 6-8 years between data collection(s). The NSHDS was started in 1985; it consists of a biobank and related survey data from about 166000 men and women in northernmost Sweden, including three population-based sub-cohorts: the Västerbotten Intervention Programme (VIP) cohort (participants aged 30, 40, 50 or 60 years), the MONICA cohort (participants aged 25-74 years), and the mammography screening cohort (women aged 50-69 years). Dietary data are available in the VIP and MONICA cohorts and are administered by the Northern Sweden Diet Database (NSDD). By December 31, 2014 Food frequency questionnaire (FFQ) data from 103256 unique individuals in the NSDDS were available for this study after exclusion of participants with incomplete food-intake data, extreme food intakes, extreme energy intakes, and implausible height and weight. Details of this selection procedure are described elsewhere (21).

All participants gave written informed consent. The NOWAC Study was approved by the Regional Committee for Medical Research Ethics and the Norwegian Data Inspectorate, 
whereas the coffee study within the NSHDS was approved by the Regional Ethical Review Board of Northern Sweden (registration number: 2013/332/31).

The NOWAC Study contributed 101320 women to the present analysis, whereas the NSHDS cohort initially contributed 103256 participants. As the questionnaires sent out during the first wave of data collection in the NOWAC Study (1991-1992) did not include questions on diet, we chose not to use the information collected at that time. We further excluded those with prevalent cancer other than non-melanoma skin cancer at baseline, those who emigrated or died before the start of follow-up ( $\mathrm{N}=8101)$, those that had missing information on coffee consumption ( $\mathrm{N}=1615)$, and those with total energy intake above 15 $000 \mathrm{~kJ}$ or below $2500 \mathrm{~kJ} / \mathrm{day}$ ( $\mathrm{N}=1362$ ). Our final sample consisted of 193439 participants (94 286 from the NOWAC Study and 99153 from NSDD), of whom 145247 (75.1 \%) were women and 48192 (24.9\%) were men.

\section{Assessment of coffee consumption and covariates}

In the NOWAC FFQ, women answered the following question on coffee consumption: "How many cups of each kind of coffee (boiled, filtered, instant) did you usually drink during the past year?' 'For each brewing method, women could choose from the following answers: never/seldom, 1-6 cups/week, 1 cup/day, 2-3 cups/day, 4-5 cups/day, 6-7 cups/day, and $\geq 8$ cups/day. In the NSHDS cohort, coffee consumption was measured as the number of occasions on which a participant consumed filtered or boiled coffee with the following alternatives: never, a few times/year, 1-3 times/month, 1 time/week, 2-3 times/week, 4-6 times/week, 1 time/day, 2-3 times/day, and >4 times/day. For the purpose of this paper, we assumed that 1 time/day from the NSHDS cohort matched 1 cup/day from the NOWAC cohort. We calculated total coffee consumption by summing the frequencies of filtered, boiled, and instant coffee in the NOWAC cohort and filtered and boiled coffee in the NSHDS 
cohort. Finally, filtered, boiled, and total coffee consumption was categorized as light consumption ( $\leq 1 \mathrm{cup} /$ day), moderate consumption ( $>1-<4$ cups/day), and heavy consumption ( $\geq 4$ cups/day).

Information on smoking status (never, former, or current), alcohol consumption, body mass index (BMI), total energy intake, and self-reported history of diabetes were also available from both cohorts.

\section{Follow-up and end points}

In the NOWAC cohort, the unique 11-digit personal number assigned to every legal resident in Norway was used to obtain information on cancer incidence through linkage to the Norwegian Cancer Registry, and to obtain information on death and emigration through linkage to the Norwegian Central Population Register. In the NSDDS cohort, the unique 12digit personal number was used to obtain information on cancer end-points through linkage to the regional branch of the Swedish Cancer Registry; mortality end-points were identified through linkage to the Swedish National Cause-of-death Registry.

The $7^{\text {th }}$ Revision of the International Statistical Classification of Diseases, Injuries and Causes of Death was used to classify bladder (181.0-181.9), esophageal (150.0-150.9), kidney (180.0-180.9), pancreatic (157.0-157.9), and stomach (151.0-151.9) cancer cases in the national and regional cancer registries.

\section{Statistical analysis}

We calculated person-years from start of follow-up until diagnosis of any incident invasive cancer other than non-melanoma skin cancer, death, emigration, or the end of the study period (31 December 2014), whichever occurred first. 
We used Cox proportional hazards regression models to calculate hazard ratios (HRs) with 95\% confidence intervals (CIs) for the association between coffee consumption and the risk of developing bladder, esophageal, kidney, pancreatic, or stomach cancer for the entire cohort and in men and women separately. Light coffee consumers ( $\leq 1 \mathrm{cup} /$ day) were used as the reference group, as the number of cases among "never/seldom” coffee consumers was low. We also believed that "never/seldom” coffee consumers could differ from the study population who reported consuming coffee more frequently, making them less appropriate as a reference group, as seldom consuming or abstaining from coffee is relatively uncommon in Scandinavia.

We used attained age as the underlying time scale. All models were stratified by study cohort. The final models for each cancer site were adjusted for a variety of the following $a$ priori selected covariates: sex, smoking status (never, former, current), body mass index (BMI, $\leq 18.49,18.5-24.9,25-29.9$, and $\geq 30 \mathrm{~kg} / \mathrm{m}^{2}$ ), alcohol consumption (0, 0.1-3.99, 4-9.99, $\geq 10 \mathrm{~g} /$ day), and self-reported history of diabetes (yes/no). In addition, the analyses of filtered coffee were adjusted for boiled coffee consumption and vice-versa.

The proportional hazards assumption was assessed by Schoenfeld residuals. To test for linear trend, a median value was assigned to each category of the ordinal coffee consumption variable, which was then modeled as a continuous variable in the analysis.

To assess a possible non-linear relationship between coffee consumption and the study outcomes, we modeled restricted cubic splines with four knots, with its locations based on Harrell's recommended percentiles of the total and filtered coffee consumption (22). The restricted cubic splines for boiled coffee were modeled with three knots, positioned at the $25^{\text {th }}$, $60^{\text {th }}$, and $95^{\text {th }}$ percentiles of the coffee distribution. We used a Wald-type test to assess if the coefficients of the second and third spline in filtered and total coffee analyses, and second spline’s coefficient in boiled coffee analyses were equal to zero. 
We assessed possible interactions between coffee consumption and BMI, as the antioxidant effects of coffee might be modified by this factor (23). We also decided to conduct analyses of all the outcomes stratified by smoking status (never/ever), as the renal clearance of caffeine was found to be different between smokers and non-smokers $(24,25)$, and a possible association between coffee consumption and the risk of bladder cancer among never smokers has been previously reported $(10,26)$.

To check for possible reverse causality, we performed analyses in which we excluded participants who were diagnosed with one of the cancers under investigation during the first year of follow-up. As part of sensitivity analyses, we assessed the effect of coffee consumption on the risk of the five cancer sites by applying Fine and Grey competing risk regression models, with all other incident cancers as competing risk (27). Finally, we conducted analyses of all of the outcomes with additional adjustment for total energy intake (kJ/day), and the analyses of pancreatic cancer in which we also adjusted for red meat intake (occasions/day).

All the analyses were done in STATA version 14.0 (Stata Corp, College Station, TX, USA).

\section{Results}

During an average of 13.6 years of follow-up and more than 2.6 million person-years, a total of 19733 cancer cases were identified, among them 479 (2.4\%) bladder cancers, 97 (0.5\%) esophageal cancers, 475 (2.5\%) kidney cancers, 491 (2.5\%) pancreatic cancers, and 281 (1.4\%) stomach cancers.

The distribution of cancer cases was similar across coffee consumption categories. Most men and women reported moderate coffee consumption. Light coffee consumers were the 
youngest at baseline, and were more likely to have been never smokers, as opposed to heavy coffee consumers, who were both the oldest and more often current smokers. (Table 1). Filtered coffee was drunk most frequently. Most of the men reported being moderate filtered coffee consumers (43.1\%), while 38.5\% of the women were light and 34.2\% were moderate filtered coffee consumers. Boiled coffee consumption was uncommon in both men and women, with $81.4 \%$ and $85.1 \%$, respectively, reporting light boiled coffee consumption. (Supplementary table 1). Moreover, 97.4\% of the heavy filtered coffee consumers were light boiled coffee consumers. On the other hand $78.9 \%$ of the heavy boiled coffee consumers reported drinking no more than 1 cup of filtered coffee per day. Overall, only 768 participants in the cohort reported drinking at least 4 cups of both filtered and boiled coffee per day (Supplementary table 2).

We did not find any evidence of an association between total coffee consumption and risk of bladder, esophageal, kidney, pancreatic, or stomach cancer in moderate, or heavy total coffee consumers compared to light total coffee consumers (Table 2). This was also true in sex-specific analyses (Table 3). Compared to light total coffee consumers, heavy total coffee consumers had a multivariable-adjusted HR of 0.83 of being diagnosed with pancreatic cancer (95\% CI 0.62-1.11). Corresponding HRs for bladder, esophageal, kidney and stomach cancers were 1.34 (95\% CI 0.94-1.90), 0.83 (95\% CI 0.42-1.61), 0.99 (95\% CI 0.74-1.33), and 0.88 (95\% CI 0.61-1.28), respectively. In addition, there was no evidence of a dose-response relationship in any of the investigated outcomes. We observed an $87 \%$ increased risk of bladder cancer (95\% CI 1.01-3.45; $p$ for trend across categories=0.03) in never smokers who were also heavy total coffee consumers compared to never smokers who were light coffee consumers.

We found a significantly reduced risk of pancreatic cancer for both moderate (HR: 0.79, 95\% CI 0.64-0.99) and heavy filtered coffee consumers (HR: 0.74, 95\% CI 0.57-0.95; $p$ for 
trend across categories=0.01) (Table 4). The association remained significant in never smokers (moderate vs. light consumers HR: 0.60, 95\% CI 0.41-0.87; heavy vs. light consumers HR: 0.60, 95\% CI 0.35-1.01; $p$ for trend across categories=0.01) (Table 7), and borderline significant in women (Table 5). We did not observe a significant association between filtered coffee consumption and risk of pancreatic cancer in men (Table 5) or in ever smokers (Table 7). Similar to a total coffee consumption, heavy filtered coffee consumption was associated with an increased risk of bladder cancer in never smokers (HR: 1.86, 95\% CI 1.12-3.10). An increased filtered coffee consumption was not associated with the risk of esophageal, kidney, or stomach cancer in the total cohort, nor was an association found in analyses stratified by gender and smoking status.

We found an increased risk of bladder cancer in women (Table 6) and in never smokers (Table 7) who were moderate boiled coffee consumers (HR: 1.58, 95\% CI 1.03-2.05; HR: 1.64, 95\% CI 1.03-2.62, respectively), with no significant trend across consumption categories ( $\mathrm{p}=0.56$ and $\mathrm{p}=0.06$, respectively). Finally, never smokers who were also heavy boiled coffee consumers had more than twice the risk of stomach cancer compared to never smokers who were light boiled coffee consumers (HR: 2.14, 95\% CI 1.10-4.16; $p$ for trend across categories $=0.04)$.

We did not observe any departure from linearity between filtered, boiled, and total intake and the study outcomes (data not shown). The associations between 1-cup/day increase in each coffee type and the risk of each of the study outcomes, estimated from the restricted cubic splines models are presented in Figures 1-3. The figure 2 show a per-cup, statistically significant, reduced risk of pancreatic cancer among those who increased their filtered coffee consumption from 2 to 6 cups/day.

The test of Schoenfeld residuals did not indicate a violation of the proportional hazards assumption. We found no evidence of effect modification between coffee consumption and 
BMI for any of the outcomes. The risk estimates for bladder, esophageal, kidney, and pancreatic cancers in analyses which did not include cancer cases diagnosed during the first year of follow-up were similar to those from the analyses that included the entire study sample. On the other hand, the estimates for stomach cancer were stronger in the analyses that excluded cases diagnosed during the first year of follow-up were excluded (data not shown). The subhazard ratios from the competing risk regression models were similar to the estimates from the ordinary Cox proportional hazards regression models (data not shown). Finally, our conclusions remained the same after the models were additionally adjusted for total energy intake and red meat consumption (data not shown).

\section{Discussion}

The data from this large joint cohort did not provide evidence of an association between total coffee consumption and the risk of esophageal, kidney, or stomach cancer. Further, no anticarcinogenic effect of diterpene rich boiled unfiltered coffee in comparison with filtered coffee was found. On the contrary, in analyses stratified by brewing technique, we found that increased filtered coffee consumption was associated with a reduced risk of pancreatic cancer. We observed an increased risk of bladder cancer for never smokers who reported consuming $\geq 4 \mathrm{cups} /$ day of total coffee, as well as an increased risk of stomach cancer for never smokers who were heavy boiled coffee consumers.

\section{Bladder cancer}

None of the significant associations we observed between coffee consumption and risk of bladder cancer are in line with the results of the study by Jacobsen et al, which included 16555 Norwegian men and women (28), and the study by Kurahashi et al, conducted among more than 100000 Japanese men and women (26). On the other hand, Wu et al found a metaodds ratio (OR) of 1.33 (95\% CI 1.19-1.48) after pooling the results from 25 case-control and 
five cohort studies. Our results for never smokers confirmed the findings of this metaanalysis, in which the authors reported a meta-OR of 1.72 (95\% CI: 1.25 to 2.35) for never smokers in the highest coffee consumption category (10). A proposed mechanism that explains why the increased risk is observed only in never smokers involves a stimulating effect of caffeine on liver enzymes CYP1A2 and NAT2. The activation of some carcinogens from cigarette smoking such as polycyclic aromatic hydrocarbons might be increased by the said enzymes $(10,26,29)$. As the metabolism of caffeine might be faster in smokers due to a shared metabolic pathway by nicotine and caffeine $(30,31)$, the potential carcinogenic effect of coffee might more pronounced in never smokers.

\section{Esophageal cancer}

Jacobsen et al found no association between coffee consumption and the risk of esophageal cancer in a study that included only 22 cases (28). In a more recent study from Japan, Naganuma et al found an inverse association between coffee consumption and the risk of oral cavity, pharyngeal, and esophageal cancers combined. They reported a 49\% decreased risk for those who drank at least 1 cup/day of coffee compared to abstainers (95\% CI 23\%$67 \%$ ), with an inverse association that was consistent regardless of sex (32). However, in a recent systematic review, Islami et al stated that there was little evidence of an association between coffee consumption and the risk of esophageal cancer after summarizing the results from 22 observational studies (12).

\section{Pancreatic cancer}

Our results regarding pancreatic cancer and total coffee consumption are in line with findings from a meta-analysis that included 15 case-control and 12 cohort studies and adjusted for smoking exposure (13). The authors reported a meta-risk ratio of 1.01 (95\% CI 0.99-1.04) for a 1-cup/day increase. Similarly, no association was found in two studies from Norway, one from Finland, or in a study from Sweden that included participants from the VIP cohort of 
the NDDS (28, 33-35). Guertin et al used data from the NIH-AARP Diet and Health cohort and did not observe an association between total, caffeinated, or decaffeinated coffee consumption and the risk of pancreatic cancer either men or women (36). The data from the VIP cohort further indicated that the consumption of $\geq 4$ cups/day of boiled coffee was associated with a 2.5-fold (95\% CI 1.15-5.50) increased risk of pancreatic cancer compared to consumption of $>1$ cup/day. The analysis, however, was severely underpowered, as the number of cases in the highest boiled coffee consumption group was only eight (34). One of the possible explanations of the difference in the association between filtered and boiled coffee and the risk of pancreatic cancer might be the difference in diet between consumers of these coffee types. Boiled coffee consumers are more likely to have more traditional food habits, and are less adapted to a modern, more sedentary lifestyle compared to filtered coffee consumers (37). This may also explain the general lack of anti-carcinogenic effect showed among consumers of boiled coffee compared with consumers of filtered coffee in this study. Increased coffee consumption has been associated with a lower risk of type 2 diabetes (38), which is an established risk factor for pancreatic cancer (39). Chlorogenic acid, one of the components of roasted coffee, was found to reduce glucose levels, thus increasing insulin sensitivity and reducing the risk of cancer, in addition to its antioxidant capacity $(7,40)$. An anticancerogenic effect of coffee diterpens, kahweol and cafestol seems unlikely based on our findings, as concentration of these compounds in filtered coffee is very small (41).

\section{Renal cancer}

Stensvold et al found no association between coffee consumption and the risk of renal cancer in a cohort of 43000 Norwegian men and women, a case-control study from Italy by Montella et al came to the same conclusion $(33,42)$. Contrary to these studies, Nilsson et al found a 70\% reduced risk of renal cancer among subjects from the VIP cohort with a total coffee consumption of $\geq 4$ cups/day compared to those who drank $>1$ cup/day. However, only 
56 cases of renal cancer were available for the VIP analysis, compared to 462 cases from the present study. The authors did not observe any preparation-specific association for filtered or boiled coffee (34).

\section{Stomach cancer}

Zeng et al found a relative risk (RR) of stomach cancer of 1.18 (95\% CI 0.90-1.15) for the highest coffee consumption category after pooling the results from 15 cohort studies, and a RR of 1.07 (95\% CI 0.95-1.21) for every 3-cup/day increase in total coffee consumption (14). No association between coffee consumption and stomach cancer was found in the studies by Jacobsen et al, Stensvold et al, or Nilsson et al, nor was an association reported in two more recent studies from Finland and Singapore (28, 33-35, 43).

\section{Strengths and limitations}

In addition to its prospective design, our study had sufficient sample size and statistical power to detect differences between coffee consumption groups in most of the studied outcomes. The high prevalence of coffee consumers in Scandinavia allowed us to explore the impact of heavy coffee consumption on the risk of cancer. Linkage to cancer registries via the unique personal identification numbers in Norway and Sweden allowed us to obtain almost complete follow-up of cancer cases.

The response rate of $52.7 \%$ of the NOWAC Study, and the participation rate of $48-79 \%$ in the sub-cohorts of the NSDDS are similar to other population-based cohorts. Both the NOWAC Study and the NSDDS have been shown to be truly population-based in validation studies, with no significant differences in lifestyle factors and incidence rates between responders and non-responders (44-47). Thus, the external validity of this study is considered to be acceptable. 
24-h dietary recall studies conducted to validate the FFQs used in the NOWAC Study and NSDDS cohorts showed a high validity of data on coffee consumption (Spearman’s correlation coefficients $r=0.82$ and $r=0.72$ respectively) (48-50). Similarly, the test-retest reliability of self-reported history of diabetes, weight, and height in these Norwegian cohorts was found to be acceptable (51-53).

Regarding brewing technique, the NOWAC Study and the NSDDS cohorts are, to the best of our knowledge, the only large population-based studies available that distinguish clearly between filtered and non-filtered coffee consumption.

There are several limitations in our study. Analyses of esophageal cancer were somewhat statistically underpowered. This also holds true for sex-specific analyses, in addition to a relatively small number of stomach cancer cases among men. Similarly, subgroup analyses by smoking status may have lacked statistical power in the analyses of bladder, esophageal and stomach cancer sites, and this problem was more pronounced in the analyses of never smokers.

We lacked information on caffeination status, as this information was not available from the questionnaires. However, as the consumption of decaffeinated coffee is uncommon in Norway and Sweden, we believe that the observed estimates reflect the association between caffeinated coffee consumption and the study outcomes (54). Similarly, total coffee consumption in the present study might have been underestimated, as we did not have information on the consumption of coffee drinks such as macchiato, espresso, cappuccino, or café latte. In addition, we lacked information on instant coffee consumption from the NSDDS cohort. However, from a chemical point of view, instant coffee is prepared by freeze-drying filtered coffee, thus this distinction is of less importance from a misclassification of brewing technique perspective. Further, consumption of these types of coffee drinks was relatively rare 
at the time of data collection. Therefore, the underestimation of total coffee consumption is likely negligible.

Even though validation studies have shown a high validity of the data on coffee consumption, misclassification is still possible, as coffee consumption was self-reported. In addition, the units of measurement of coffee consumption were different between the cohorts (occasions/day vs. cups/day), and there may have been individual variation in cup size as well. Given this, there is a potential for misclassification in total coffee consumption as a consequence of adding up categorical variables; however, even if misclassification is present, it is most likely non-differential.

Finally, residual confounding of some factors cannot be ruled out. For example, we did not adjust for tea consumption, as this information was not available from the NOWAC Study questionnaire. Additional adjustment for tea consumption could have attenuated the observed associations, as tea and coffee share some biologically active compounds.

In conclusion, based on the data from the NOWAC and NSDDS cohorts, an increased consumption of filtered coffee was associated with a lower risk of pancreatic cancer, and this effect was more pronounced in never smokers. No association was found between coffee consumption and the risk of esophageal or kidney cancer. The significantly increased risk of bladder and stomach cancer we observed was confined to never smokers, although the analyses were hampered by the small number of cases.

Sources of funding: Supported by UiT The Arctic University of Norway. The funding sources had no involvement in the design and conduct of the study; the collection, management, analysis, or interpretation of the data; the preparation, review, or approval of the manuscript; or the decision to submit the manuscript for publication. 
Conflicts of interest: The authors declare that they have no conflict of interest. Disclaimer: Some of the data in this article are from the Cancer Registry of Norway. The Cancer Registry of Norway is not responsible for the analysis or interpretation of the data presented.

Acknowledgements: The authors thank the NOWAC Study staff and participants for their contribution. The authors used the services of Trudy Perdix-Thoma for the language editing of the manuscript. We acknowledge the Northern Sweden Diet Database and the funds supporting it, including the Swedish Research Council (VR), the Swedish Research Council for Health, Working Life and Welfare (FORTE) and the Västerbotten County Council.

\section{References}

1. Bohn SK, Blomhoff R, Paur I. Coffee and cancer risk, epidemiological evidence, and molecular mechanisms. Mol Nutr Food Res. 2014;58(5):915-30.

2. Gasscht F, Dicato M, Diederich M. Coffee provides a natural multitarget pharmacopeia against the hallmarks of cancer. Genes Nutr. 2015;10(6).

3. Majer BJ, Hofer E, Cavin C, Lhoste E, Uhl M, Glatt HR, et al. Coffee diterpenes prevent the genotoxic effects of 2-amino-1-methyl-6-phenylimidazo[4,5-b]pyridine (PhIP) and Nnitrosodimethylamine in a human derived liver cell line (HepG2). Food Chem Toxicol. 2005;43(3):43341.

4. Shearer J, Farah A, de Paulis T, Bracy DP, Pencek RR, Graham TE, et al. Quinides of roasted coffee enhance insulin action in conscious rats. J Nutr. 2003;133(11):3529-32.

5. Higgins LG, Cavin C, Itoh K, Yamamoto M, Hayes JD. Induction of cancer chemopreventive enzymes by coffee is mediated by transcription factor Nrf2. Evidence that the coffee-specific diterpenes cafestol and kahweol confer protection against acrolein. Toxicol Appl Pharmacol. 2008;226(3):328-37.

6. Oh JH, Lee JT, Yang ES, Chang JS, Lee DS, Kim SH, et al. The coffee diterpene kahweol induces apoptosis in human leukemia U937 cells through down-regulation of Akt phosphorylation and activation of JNK. Apoptosis. 2009;14(11):1378-86.

7. Renehan AG, Roberts DL, Dive C. Obesity and cancer: pathophysiological and biological mechanisms. Arch Physiol Biochem. 2008;114(1):71-83.

8. Kotsopoulos J, Eliassen AH, Missmer SA, Hankinson SE, Tworoger SS. Relationship between caffeine intake and plasma sex hormone concentrations in premenopausal and postmenopausal women. Cancer. 2009;115(12):2765-74.

9. Urgert R, Katan MB. The cholesterol-raising factor from coffee beans. Annu Rev Nutr. 1997; 17:305-24.

10. Wu WX, Tong YQ, Zhao Q, Yu GX, Wei XY, Lu Q. Coffee consumption and bladder cancer: a meta-analysis of observational studies. Sci Rep-Uk. 2015;5. 
11. Loomis D, Guyton KZ, Grosse Y, Lauby-Secretan B, El Ghissassi F, Bouvard V, et al. Carcinogenicity of drinking coffee, mate, and very hot beverages. Lancet Oncol. 2016;17(7):877-8. 12. Islami F, Boffetta P, Ren JS, Pedoeim L, Khatib D, Kamangar F. High-temperature beverages and foods and esophageal cancer risk--a systematic review. Int J Cancer. 2009;125(3):491-524.

13. Turati F, Galeone C, Edefonti V, Ferraroni M, Lagiou P, La Vecchia C, et al. A meta-analysis of coffee consumption and pancreatic cancer. Ann Oncol. 2012;23(2):311-8.

14. Zeng SB, Weng $\mathrm{H}$, Zhou M, Duan XL, Shen XF, Zeng XT. Long-Term Coffee Consumption and Risk of Gastric Cancer A PRISMA-Compliant Dose-Response Meta-Analysis of Prospective Cohort Studies. Medicine. 2015;94(38).

15. World Cancer Research Fund International/American Institute for Cancer Research. Continuous Update Project Report: Diet, Nutrition, Physical Activity and Kidney Cancer. 2015. Available at: wcrf.org/kidney-cancer-2015

16. World Cancer Research Fund International/American Institute for Cancer Research. Continuous Update Project Report: Food, Nutrition, Physical Activity and the Prevention of Pancreatic Cancer. 2012. Available at: http://www.dietandcancerreport.org

17. International Coffee Council. Trends in coffee consumption in selected importing countries [Internet]. London, UK: International Coffee Organization. 2012. Available from:

http://www.ico.org/documents/icc-109-8e-trends-consumption.pdf

18. Ferlay J, Soerjomataram I, Dikshit R, Eser S, Mathers C, Rebelo M, et al. Cancer incidence and mortality worldwide: sources, methods and major patterns in GLOBOCAN 2012. Int J Cancer. 2015;136(5):E359-86.

19. Lund E, Dumeaux V, Braaten T, Hjartaker A, Engeset D, Skeie G, et al. Cohort profile: The Norwegian Women and Cancer Study--NOWAC--Kvinner og kreft. Int J Epidemiol. 2008;37(1):36-41.

20. Hallmans G, Agren A, Johansson G, Johansson A, Stegmayr B, Jansson JH, et al. Cardiovascular disease and diabetes in the Northern Sweden Health and Disease Study Cohort - evaluation of risk factors and their interactions. Scand J Public Health Suppl. 2003;61:18-24.

21. Tognon G, Nilsson LM, Shungin D, Lissner L, Jansson JH, Renstrom F, et al. Nonfermented milk and other dairy products: associations with all-cause mortality. Am J Clin Nutr. 2017;105(6):1502-11.

22. Jr. HFE. Regression Modeling Strategies: With Applications to Linear Models, Logistic Regression, and Survival Analysis. New York: Springer; 2001.

23. Bakuradze T, Boehm N, Janzowski C, Lang R, Hofmann T, Stockis JP, et al. Antioxidant-rich coffee reduces DNA damage, elevates glutathione status and contributes to weight control: results from an intervention study. Mol Nutr Food Res. 2011;55(5):793-7.

24. Parsons WD, Neims AH. Effect of smoking on caffeine clearance. Clin Pharmacol Ther. 1978;24(1):40-5.

25. Campbell ME, Spielberg SP, Kalow W. A urinary metabolite ratio that reflects systemic caffeine clearance. Clin Pharmacol Ther. 1987;42(2):157-65.

26. Kurahashi N, Inoue M, Iwasaki M, Sasazuki S, Tsugane S, Japan Public Health Center Study G. Coffee, green tea, and caffeine consumption and subsequent risk of bladder cancer in relation to smoking status: a prospective study in Japan. Cancer Sci. 2009;100(2):294-91.

27. Fine JP, Gray RJ. A proportional hazards model for the subdistribution of a competing risk. J Am Stat Assoc. 1999;94(446):496-509.

28. Jacobsen BK, Bjelke E, Kvale G, Heuch I. Coffee drinking, mortality, and cancer incidence: results from a Norwegian prospective study. J Natl Cancer Inst. 1986;76(5):823-31.

29. Porta M, Vioque J, Ayude D, Alguacil J, Jariod M, Ruiz L, et al. Coffee drinking: the rationale for treating it as a potential effect modifier of carcinogenic exposures. Eur J Epidemiol. 2003;18(4):289-98.

30. Hukkanen J, Jacob P, 3rd, Peng M, Dempsey D, Benowitz NL. Effect of nicotine on cytochrome P450 1A2 activity. Br J Clin Pharmacol. 2011;72(5):836-8.

31. Gu L, Gonzalez FJ, Kalow W, Tang BK. Biotransformation of caffeine, paraxanthine, theobromine and theophylline by CDNA-expressed human CYP1A2 and CYP2E1. Pharmacogenetics. 1992;2(2):73-7. 
32. Naganuma T, Kuriyama S, Kakizaki M, Sone T, Nakaya N, Ohmori-Matsuda K, et al. Coffee consumption and the risk of oral, pharyngeal, and esophageal cancers in Japan: the Miyagi Cohort Study. Am J Epidemiol. 2008;168(12):1425-32.

33. Stensvold I, Jacobsen BK. Coffee and cancer: a prospective study of 43,000 Norwegian men and women. Cancer Causes Control. 1994;5(5):401-8.

34. Nilsson LM, Johansson I, Lenner P, Lindahl B, Van Guelpen B. Consumption of filtered and boiled coffee and the risk of incident cancer: a prospective cohort study. Cancer Causes Control. 2010;21(10):1533-44.

35. Bidel S, Hu G, Jousilahti P, Pukkala E, Hakulinen T, Tuomilehto J. Coffee consumption and risk of gastric and pancreatic cancer--a prospective cohort study. Int J Cancer. 2013;132(7):1651-9.

36. Guertin KA, Freedman ND, Loftfield E, Stolzenberg-Solomon RZ, Graubard BI, Sinha R. A prospective study of coffee intake and pancreatic cancer: results from the NIH-AARP Diet and Health Study. Brit J Cancer. 2015;113(7):1081-5.

37. Nilsson ML. Chapter 29-Boiled Coffee: An Arctic Example of Potential Residual and Unmeasured Confounding in Coffee Epidemiology. In: Preedy V, editor. Coffee in Health and Disease Prevention. Massachusetts: Academic Press; 2014. p. 265-74.

38. Ding M, Bhupathiraju SN, Chen M, van Dam RM, Hu FB. Caffeinated and decaffeinated coffee consumption and risk of type 2 diabetes: a systematic review and a dose-response meta-analysis. Diabetes Care. 2014;37(2):569-86.

39. Bosetti C, Rosato V, Li D, Silverman D, Petersen GM, Bracci PM, et al. Diabetes, antidiabetic medications, and pancreatic cancer risk: an analysis from the International Pancreatic Cancer CaseControl Consortium. Ann Oncol. 2014;25(10):2065-72.

40. Shearer J, Farah A, de Paulis T, Bracy DP, Pencek RR, Graham TE, et al. Quinides of roasted coffee enhance insulin action in conscious rats. J Nutr. 2003;133(11):3529-32.

41. Parras P, Martinez-Tome M, Jimenez AM, Murcia MA. Antioxidant capacity of coffees of several origins brewed following three different procedures. Food Chem. 2007;102(3):582-92.

42. Montella M, Tramacere I, Tavani A, Gallus S, Crispo A, Talamini R, et al. Coffee, Decaffeinated Coffee, Tea Intake, and Risk of Renal Cell Cancer. Nutr Cancer. 2009;61(1):76-80.

43. Ainslie-Waldman CE, Koh WP, Jin A, Yeoh KG, Zhu F, Wang R, et al. Coffee intake and gastric cancer risk: the Singapore Chinese health study. Cancer Epidemiol Biomarkers Prev. 2014;23(4):63847.

44. Lund E, Kumle M, Braaten T, Hjartaker A, Bakken K, Eggen E, et al. External validity in a population-based national prospective study--the Norwegian Women and Cancer Study (NOWAC). Cancer Causes Control. 2003;14(10):1001-8.

45. Stegmayr B, Lundberg $V$, Asplund $K$. The events registration and survey procedures in the Northern Sweden MONICA Project. Scand J Public Health Suppl. 2003;61:9-17.

46. Pukkala E, Andersen A, Berglund G, Gislefoss R, Gudnason V, Hallmans G, et al. Nordic biological specimen banks as basis for studies of cancer causes and control--more than 2 million sample donors, 25 million person years and 100,000 prospective cancers. Acta Oncol. 2007;46(3):286-307.

47. Weinehall L, Hallgren CG, Westman G, Janlert U, Wall S. Reduction of selection bias in primary prevention of cardiovascular disease through involvement of primary health care. Scand $J$ Prim Health Care. 1998;16(3):171-6.

48. Parr CL, Veierod MB, Laake P, Lund E, Hjartaker A. Test-retest reproducibility of a food frequency questionnaire (FFQ) and estimated effects on disease risk in the Norwegian Women and Cancer Study (NOWAC). Nutr J. 2006;5:4.

49. Hjartaker A, Andersen LF, Lund E. Comparison of diet measures from a food-frequency questionnaire with measures from repeated 24-hour dietary recalls. The Norwegian Women and Cancer Study. Public Health Nutr. 2007;10(10):1094-103.

50. Johansson I, Hallmans G, Wikman A, Biessy C, Riboli E, Kaaks R. Validation and calibration of food-frequency questionnaire measurements in the Northern Sweden Health and Disease cohort. Public Health Nutrition. 2002;5(3):487-96. 
51. Sheikh MA, Lund E, Braaten T. Test-retest reliability of self-reported diabetes diagnosis in the Norwegian Women and Cancer Study: A population-based longitudinal study $(n=33,919)$. SAGE Open Med. 2016;4:2050312115622857.

52. Skeie G, Mode N, Henningsen M, Borch KB. Validity of self-reported body mass index among middle-aged participants in the Norwegian Women and Cancer study. Clin Epidemiol. 2015;7:313-23. 53. Rylander C, Sandanger TM, Engeset D, Lund E. Consumption of lean fish reduces the risk of type 2 diabetes mellitus: a prospective population based cohort study of Norwegian women. PLoS One. 2014;9(2):e89845.

54. Statistik Sentralbyrå Informasjonstjeneste [Internet]. Uten kaffe stopper Norge. Statistics Norway. 2011 [cited 15 May 2017]. Available from: https://www.ssb.no/utenriksokonomi/artiklerog-publikasjoner/uten-kaffe-stopper-norge 